\title{
Polymorphism analysis of IGFBP-5 gene exon 1 in Tibet Mini-pig and Junmu No. 1 White pig
}

\author{
A.D. Chen' ${ }^{1}$ L.L. Hao ${ }^{2}$, X.B. Fang ${ }^{2}$, K. Lu' ${ }^{2}$, S.C. Liu ${ }^{2}$ and Y.L. Zhang ${ }^{1}$ \\ ${ }^{1}$ Animal Nutrition Department, College of Animal Science, \\ South China Agricultural University, Guangzhou, Guangdong Province, China \\ ${ }^{2}$ Animal Biotechnology Department, Jinlin University, Changchun, \\ Jilin Province, China \\ Corresponding authors: Y.L. Zhang / L.L. Hao \\ E-mail: zhangyl@scau.edu.cn / haolinlin@jlu.edu.cn
}

Genet. Mol. Res. 13 (1): 1643-1649 (2014)

Received September 10, 2013

Accepted February 12, 2013

Published March 12, 2014

DOI http://dx.doi.org/10.4238/2014.March.12.17

\begin{abstract}
The genetic resources and the mechanism of miniaturization in the Tibet Mini-pig have not been comprehensively studied. Polymorphisms in genes related to the insulin-like growth factor (IGF) axis have been investigated for years, but few on the polymorphism of IGF-binding protein-5 (IGFBP-5) in the Tibetan pig. In this study, allele-specific polymerase chain reaction (AS-PCR) was used to analyze polymorphisms in exon 1 of the IGFBP-5 gene in two pig breeds, Tibet Mini-pigs and Junmu No. 1 White pigs. A BLAST analysis of the expressed sequence tags in the porcine IGFBP-5 gene revealed that exon 1 of this gene has two single nucleotide polymorphisms (SNPs), G188T and G503A. The AS-PCR results demonstrated that in both pig breeds examined, the TT, GT, and GG genotypes existed at the G188T locus, with GT as the most common genotype. At the G503A locus, GG, GA, and AA genotypes existed in Junmu No. 1 White pigs, with the GA genotype as the most frequently
\end{abstract}


occurring. By contrast, at this locus, only the GA and AA genotypes were observed in the Tibetan pigs, and AA was more common than GA. There was a significant difference $(\mathrm{P}<0.01)$ in allele distribution between the two breeds at the G503A locus but not the G188T locus, and there was a lower polymorphism information content for the two polymorphic loci in Tibet Mini-pigs than in Junmu No. 1 White pigs. The present study revealed SNPs in exon 1 of IGFBP-5 gene in the Tibet Mini-pig, possibly providing more understanding of the mechanism of miniaturization.

Key words: Pigs; IGFBP-5 gene; AS-PCR (allele-specific PCR); SNP

\section{INTRODUCTION}

The full-length insulin-like growth factor-binding protein-5 (IGFBP-5) molecule is a secreted protein that contains 252 amino acid residues and has a molecular weight of 29 $\mathrm{kDa}$ (Beattie et al., 2006). IGFBP-5 is an important member of the IGF axis that exists in blood and in various tissues. IGFBP-5 has the highest affinity for IGFs among the IGFBPs and stabilizes IGF concentration in serum and regulates IGF function (Clemmons, 1993). In addition, IGFBP-5 can bind to hydroxyapatite in bone extracellular matrix to stimulate IGF increases in bone, thereby promoting the proliferation of osteoblasts (Mohan, 1993; Kanzaki et al., 1994; Conover, 1995; Mohan et al., 1995a,b). The ways in which polymorphisms in genes related to the IGF axis affect porcine growth and development have been investigated for years both in China and around the world; however, few studies have reported the effects of IGFBP-5 on the regulation of porcine growth. A study by Zhao et al. (2009) has demonstrated that this gene may influence porcine growth by regulating early serum hormone levels. Some associations of IGFBP-5 single nucleotide polymorphisms (SNPs) with pig production traits have been reported. For example, Fan et al. (2009) reported that IGFBP-5 genes are associated with fat deposition traits in the pig. Sironen et al. (2010) found effects of polymorphisms in $I G F B P-5$ genes on reproduction traits in Finnish pig populations.

The Tibetan pig is a small pig that is a unique breed in China. This breed originated in semi-agricultural and semi-pastoral areas of the Qinghai-Tibet Plateau, which features altitudes of 2500-4300 $\mathrm{m}$ above sea level (Li and Luo, 1993). The Tibetan pig is relatively small and grows slowly, but is a genetically valuable resource for the pig species, because they exhibit various desirable characteristics, including the presence of a high percentage of lean meat, more intramuscular fat than other pig species, and tender meat with a rich, wild flavor (Fan et al., 2003). The Junmu No. 1 White pig is a large breed of pig that demonstrates rapid growth, high meat production rates, good fecundity, and strong adaptability with respect to disease resistance (Gao et al., 2011). Therefore, the study of polymorphisms in the IGFBP-5 gene in Tibetan pigs and Junmu No. 1 White pigs should be conducive to understanding the breeding and growth mechanisms of pigs. In this study, we used allelespecific polymerase chain reaction (AS-PCR) to detect polymorphisms in Tibetan pigs and Junmu No. 1 White pigs at two potential SNPs in the IGFBP-5 gene. In addition, the relationship between the IGFBP-5 genotype frequencies and the porcine body length trait was investigated. 


\section{MATERIAL AND METHODS}

\section{Experimental materials}

In total, 52 Tibetan pigs were purchased from the Beijing Tonghe Shengtai Institute of Comparative Medicine, and 30 Junmu No. 1 White pigs were purchased from the original breeding farm for this breed, which is administered by the College of Animal Science and Veterinary Medicine of Jilin University. Approximately $2.0 \mathrm{~g}$ liver tissue were collected from each pig and placed in a sterile $1.5-\mathrm{mL}$ centrifuge tube, which was transported to the laboratory in an ice box. These tissue samples were then stored at $-80^{\circ} \mathrm{C}$ for later use. Animal experiments were done in accordance with the guidelines on animal care and use established by the Jilin University Animal Care and Use Committee.

\section{Extraction of genomic DNA}

In accordance with the manufacturer instructions, genomic DNA was obtained from the collected liver tissue samples using a spin-column cell/tissue genomic DNA extraction kit purchased from BioTeke Corporation (Beijing, China). Product purity was assessed by measuring the ratio of the optical density of 260 and $280 \mathrm{~nm}$, which was typically between 1.7 and 1.9. The product was then stored at $-20^{\circ} \mathrm{C}$ for later assays.

\section{Design of allele-specific primers}

The Oligo 6.0 and Primer 5.0 software programs were utilized to design allele-specific primers in accordance with the porcine IGFBP-5 sequences provided by expressed sequence tags and the principles of AS-PCR (Bottema and Sommer, 1993). A pair of allele-specific primers was designed for each mutation locus (Table 1). Primers BP188A and BP188C were used to detect mutations in G188T, whereas primers BP503C and BP503T were used to detect mutations in G503A. These primers were synthesized by the Beijing Genomics Institute, diluted with sterile deionized water, and stored at $-20^{\circ} \mathrm{C}$.

\begin{tabular}{lllc}
\multicolumn{4}{c}{ Table 1. AS-PCR primers for exon 1 of the porcine $I G F B P-5$ gene. } \\
\hline Name & Direction & Primer sequence $\left(5^{\prime}-3^{\prime}\right)$ & Product (bp) \\
\hline BP188A & Forward & CCTTTCTCCTTGCACTCTCGA & 210 \\
& Reverse & GCTCCTTGACCAGCTCACAG & 326 \\
BP188C & Forward & CCTTTCTCCTTCACTCTCGC & 225 \\
& Reverse & CGGCTTCTCCTCGTCCTG & 297 \\
BP503C & Forward & GAGAGCCGCTGCACGCC & 29 \\
& Reverse & TTCCAAGGTGCAAGGACCTC & \\
BP503T & Forward & GAGAAGCCGCTGCACGCT & \\
& Reverse & GAGAAGGAGCGAAACCTGCAG & \\
\hline
\end{tabular}

\section{AS-PCR}

The PCR amplification system included the following components: $1.5 \mu \mathrm{L}$ template genomic DNA $(25 \mu \mathrm{g} / \mathrm{mL}), 1 \mu \mathrm{L} 10 \mu \mathrm{M}$ of each forward and reverse primer, $10 \mu \mathrm{L} 10 \mathrm{X}$ Ex Taq buffer ( $\mathrm{Mg}^{2+}$-free), $2.5 \mu \mathrm{L}$ Ex Taq ${ }^{\mathrm{TM}}$ polymerase $(5 \mathrm{U} / \mu \mathrm{L}), 2 \mu \mathrm{L}$ dNTPs $(2.5 \mu \mathrm{M})$, and ultra- 
pure water up to a total reaction volume of $25 \mu \mathrm{L}$. The following reaction conditions were employed: denaturation at $94^{\circ} \mathrm{C}$ for $5 \mathrm{~min} ; 30$ cycles of $95^{\circ} \mathrm{C}$ for $30 \mathrm{~s}, 59^{\circ} \mathrm{C}$ for $20 \mathrm{~s}$, and $72^{\circ} \mathrm{C}$ for $30 \mathrm{~s}$; and extension at $72^{\circ} \mathrm{C}$ for $8 \mathrm{~min}$. The reaction products were separated by $2 \%$ agarose gel electrophoresis, and a gel imaging system was used to observe the electrophoretic bands.

A total of $1 \mu \mathrm{L}$ of each porcine genomic DNA template obtained was mixed with a 95$\mu \mathrm{L}$ gene pool. To detect the quality of the allele-specific primers, this pooled DNA was used as a template for PCR amplification.

\section{Genotype determination}

Genotype determinations were based on the band patterns of the PCR products after $2 \%$ agarose gel electrophoresis. In particular, $I G F B P-5$ genotypes were screened and identified if PCR with an allele-specific primer pair generated the amplification product of one genotype but no amplification product for another genotype.

\section{Statistical analysis}

Liver tissue samples from 82 healthy experimental pigs were assayed using the allele-specific primers under optimized reaction conditions. Allele frequencies, genotype frequencies, and the heterozygosities of the SNPs examined were statistically analyzed and determined. Polymorphic information content (PIC) was determined using the PIC_Calc 0.6 software program (http://www.seekbio.com/soft/2045.html).

\section{RESULTS}

\section{Allele-specific primer specificity tests}

PCR amplifications using the pooled DNA as the template material demonstrated that all primers tested gave a specific band. These results agreed with theoretical expectations. Furthermore, no non-specific bands were observed (Figure 1), indicating that the four pairs of allele-specific primers exhibited satisfactory specificity.

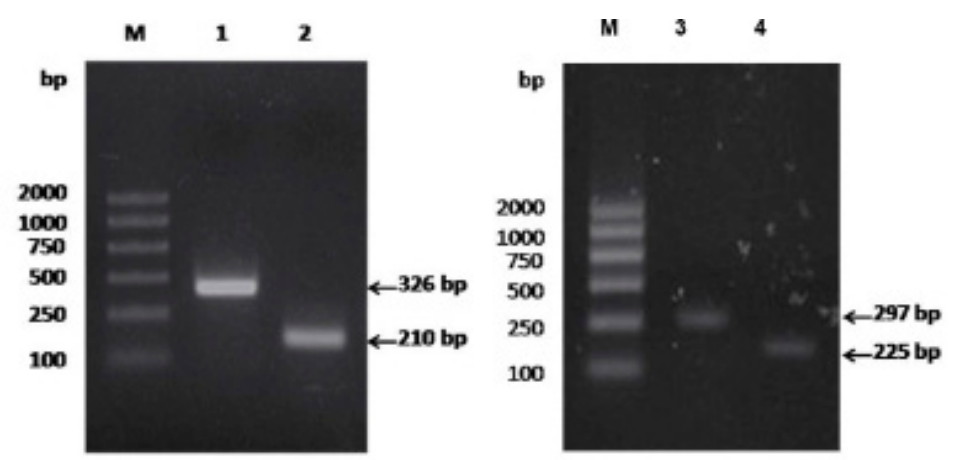

Figure 1. Determinations of primer specificity. Lane $M=$ DL2000 marker; lane $1=$ BP188C PCR amplification product $(326 \mathrm{bp})$; lane $2=\mathrm{BP} 188 \mathrm{~A}$ PCR amplification product $(210 \mathrm{bp})$; lane $3=\mathrm{BP} 503 \mathrm{~T}$ PCR amplification product (297 bp); lane $4=$ BP503C PCR amplification product (225 bp). 


\section{AS-PCR analysis of genotypes}

Using allele-specific primers under the optimized reaction conditions, AS-PCR was employed to amplify and assess all genomic DNA samples from the experimental populations of the two pig breeds examined. Variations and genotypes were determined in accordance with the parallel amplification products generated by PCR with each primer pair (Figure 2). For instance, samples J1, J3, J6-J7, and J14-J15 generated PCR products with both the BP503T and BP503C primer pairs, indicating a 503G/A heterozygous genotype. Samples J4-J5, J8-J9 and J13 generated PCR products with the BP503T primer pair but not the BP503C primer pair, indicating a 503A/A homozygous genotype. Samples J2 and J10-J12 generated PCR products with the BP503C primer pair but not the BP503T primer pair, indicating a 503G/G homozygous genotype.

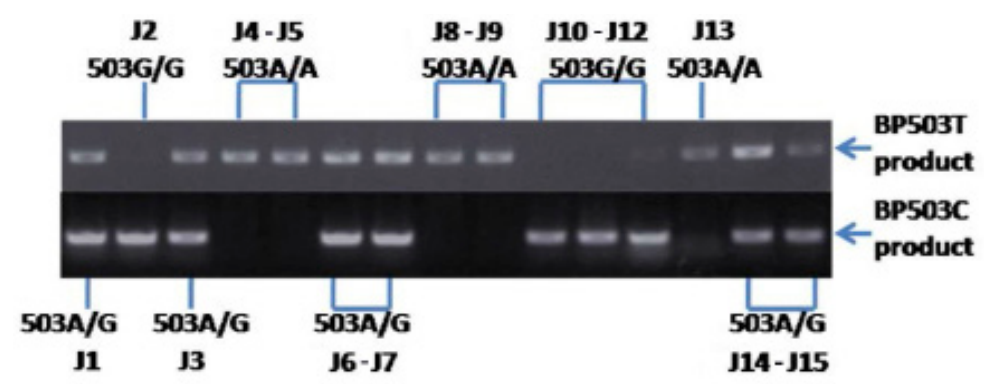

Figure 2. Analysis of the AS-PCR products by agarose gel electrophoresis.

\section{Statistical analysis of allele and genotype frequencies}

Allele and genotype frequencies were statistically analyzed (Table 2), and PIC was assessed for the two breeds examined (Table 3). As indicated in Tables 2 and 3, in both pig breeds, the TT, GT, and GG genotypes existed at the G188T locus of exon 1 of the IGFBP-5 gene, with GT as the predominant genotype. At this locus, T was the dominant allele, and the PIC values ranged from 0.42 to 0.48 ; there were no significant differences in genotype distribution between the two pig breeds $(\mathrm{P}>0.05)$. At the G503A locus of exon 1, the GG, GA, and AA genotypes existed in Junmu No. 1 White pigs, with GA as the predominant genotype. By contrast, only the GA and AA genotypes existed at this locus in Tibetan pigs, and the homozygous AA genotype was more common than the GA genotype. A was the dominant allele at the G503A locus in both breeds. The PIC values of this locus ranged from 0.35 to 0.54 , and the two breeds exhibited significantly different genotype distributions at this locus $(\mathrm{P}<0.01)$. The PIC values of the two loci were lower for Tibet pigs than for Junmu No. 1 White pigs.

\begin{tabular}{|c|c|c|c|c|c|c|c|c|c|c|c|c|c|c|c|}
\hline \multirow[t]{3}{*}{ Population } & \multirow[t]{3}{*}{$\begin{array}{l}\text { Number of } \\
\text { individuals }\end{array}$} & \multicolumn{6}{|c|}{$\begin{array}{l}\text { Genotype frequency (\%) } \\
\text { number of individuals }\end{array}$} & \multicolumn{4}{|c|}{ Allele frequency (\%) } & \multicolumn{2}{|c|}{$\chi^{2}$} & \multicolumn{2}{|c|}{$\mathrm{P}$} \\
\hline & & \multicolumn{3}{|c|}{188} & \multicolumn{3}{|c|}{503} & \multicolumn{2}{|c|}{188} & \multicolumn{2}{|c|}{503} & \multirow[b]{2}{*}{188} & \multirow[b]{2}{*}{503} & \multirow[b]{2}{*}{188} & \multirow[b]{2}{*}{503} \\
\hline & & TT & TG & GG & $\mathrm{AA}$ & GA & GG & $\mathrm{T}$ & G & A & G & & & & \\
\hline Tibet Mini-pigs & 52 & $15(8)$ & $70(36)$ & $15(8)$ & $65(34)$ & $35(18)$ & $0(0)$ & 50 & 50 & 82.5 & 17.5 & 4.341 & $39.38^{*}$ & 0.1141 & $<0.01$ \\
\hline $\begin{array}{r}\text { Junmu No. } 1 \\
\text { White pigs }\end{array}$ & 30 & $27(8)$ & $60(18)$ & $13(4)$ & $27(8)$ & $53(16)$ & $20(6)$ & 57 & 43 & 53.5 & 46.5 & & & & \\
\hline
\end{tabular}


Table 3. PIC values in exon 1 of the IGFBP-5 gene in Tibet Mini-pigs and Junmu No. 1 White pigs.

\begin{tabular}{lcc}
\hline Population & SNP locus & PIC value \\
\hline Tibet Mini-pigs & 188 & 0.4198875 \\
& 503 & 0.3514875 \\
Junmu No. 1 White pigs & 188 & 0.48307998 \\
& 503 & 0.53694078 \\
\hline
\end{tabular}

\section{DISCUSSION}

The AS-PCR results demonstrated that in exon 1 of the porcine IGFBP-5 gene, there are two SNPs, which define a total of six genotypes. In Tibet Mini-pigs, the GG homozygous genotype was not observed at the G503A locus of this exon. This phenomenon reflected the small number of individuals in the Tibet Mini-pig population; in particular, the high degree of inbreeding caused by the main breeding approaches of closed-colony breeding and direct inbreeding has led to the absence of a particular genotype. Thus, it is not surprising that the analysis of the genotypes at the G503A locus of the two porcine populations examined with the chi-square test for independence revealed that the genotype distributions at this SNP locus were significantly different between the two breeds $(\mathrm{P}<0.01)$. This finding is somewhat reflective of the large difference in genetic backgrounds between Tibet Mini-pigs and Junmu No. 1 White pigs. The genetic characteristics of different pig breeds were formed through the continued implementation of breeding initiatives by various human populations under particular natural and social conditions. The polymorphisms present in a breed are mainly based on the breed's genetic basis. More extensive genetic bases are associated with more abundant gene polymorphisms. The Tibet Mini-pig population used in this study is a small unique breed in China. Because these pigs are found on a remote plateau, little interbreeding between Tibetan pigs and other pig breeds has occurred. The resulting inbreeding within the limited population of Tibetan pigs has most likely led to a lack of certain alleles in the Tibetan pig gene pool. By contrast, Junmu No. 1 White pigs are local pigs raised in China with ancestry that includes contributions from several European breeds (Dabai, Changbai, etc.); therefore, Junmu No. 1 White pigs have more abundant polymorphic alleles than Tibet Mini-pigs do.

In exon 1 of the porcine $I G F B P-5$ gene, the populations studied exhibited little variation in PIC values at the G188T locus, which ranged from 0.42 to 0.49 , indicating moderate polymorphism in both Tibet Mini-pigs and Junmu No. 1 White pigs. At the G503A locus, there was larger PIC variation between the populations examined, as these values generally ranged from 0.35 to 0.54 , indicating the presence of moderate polymorphism among Tibet Mini-pigs and high polymorphism among Junmu No. 1 White pigs (Bottema and Sommer, 1993). In addition, at both SNP loci examined, PIC values were lower for Tibet Mini-pigs than Junmu No. 1 White pigs. Accordingly, the extent of genetic variation at these loci was lower for the Tibet Mini-pig population than for the Junmu No. 1 pig population, suggesting that the body length of Tibet Mini-pigs may have resulted from inbreeding.

Because IGFBP-5 exhibits the highest affinity for IGFs among the IGFBPs, IGFBP-5 was reported to enhance IGF expression and control the half-life of IGFs (Schneider et al., 2002); thus, IGFBP-5 may affect the growth traits of pigs. The results of this study indicate that polymorphisms exist in exon 1 of the $I G F B P-5$ gene, and that there are significant differences in polymorphism distributions between the two pig populations examined. Given these 
results, the effects of the gene polymorphisms studied on various pig performance characteristics (such as water retention capacity, flesh color and brightness, and hind leg muscle mass) should be examined in future investigations.

\section{ACKNOWLEDGMENTS}

Research supported by the National Natural Science Foundation (\#31272529, \#31101781 and \#30871839) and National Basic Research Program of China (“973” program) (\#2013CB127304).

\section{REFERENCES}

Beattie J, Allan GJ, Lochrie JD and Flint DJ (2006). Insulin-like growth factor-binding protein-5 (IGFBP-5): a critical member of the IGF axis. Biochem. J. 395: 1-19.

Bottema CD and Sommer SS (1993). PCR amplification of specific alleles: rapid detection of known mutations and polymorphisms. Mutat. Res. 288: 93-102.

Clemmons DR (1993). IGF binding proteins and their functions. Mol. Reprod. Dev. 35: 368-374.

Conover CA (1995). Insulin-like growth factor binding protein proteolysis in bone cell models. Prog. Growth Factor Res. 6: 301-309.

Fan B, Yang SL, Liu B, Yu M, et al. (2003). Characterization of the genetic diversity on natural populations of Chinese miniature pig breeds. Anim. Genet. 34: 465-466.

Fan B, Onteru SK and Rothschild MF (2009). The GGT1 and IGFBP5 genes are associated with fat deposition traits in the pig (Brief Report). Arch. Tierz. 52: 337-339.

Gao Y, Zhang YH, Zhang S, Li F, et al. (2011). Association of A-FABP gene polymorphism in intron 1 with meat quality traits in Junmu No. 1 white swine. Gene 487: 170-173.

Kanzaki S, Hilliker S, Baylink DJ and Mohan S (1994). Evidence that human bone cells in culture produce insulin-like growth factor-binding protein-4 and -5 proteases. Endocrinology 134: 383-392.

Li JY and Luo Z (1993). A Research on the habits and characteristics of Tibet pigs on Tibet plateau. Ecol. Domestic Anim. 14: $18-21$.

Mohan S (1993). Insulin-like growth factor binding proteins in bone cell regulation. Growth Regul. 3: 67-70.

Mohan S, Farley JR and Baylink DJ (1995a). Age-related changes in IGFBP-4 and IGFBP-5 levels in human serum and bone: implications for bone loss with aging. Prog. Growth Factor Res. 6: 465-473.

Mohan S, Nakao Y, Honda Y, Landale E, et al. (1995b). Studies on the mechanisms by which insulin-like growth factor (IGF) binding protein-4 (IGFBP-4) and IGFBP-5 modulate IGF actions in bone cells. J. Biol. Chem. 270: 2042420431.

Schneider MR, Wolf E, Hoeflich A and Lahm H (2002). IGF-binding protein-5: flexible player in the IGF system and effector on its own. J. Endocrinol. 172: 423-440.

Sironen AI, Uimari P, Serenius T, Mote B, et al. (2010). Effect of polymorphisms in candidate genes on reproduction traits in Finnish pig populations. J. Anim. Sci. 88: 821-827.

Zhao X-F, Ou Y-K, Zhang Y and Xu N-Y (2009). Quick screening SNP on partial coding sequence of insulin-like growth factor binding protein 5 gene (IGFBP5) and effect of its polymorphism on production performance in pigs. J. Agric. Biotechnol. 17: 758-762. 